\title{
Diagnostic value of diffusion-weighted MR imaging in thyroid disease: application in differentiating benign from malignant disease
}

Yingwei $\mathrm{Wu}^{1+}$, Xiuhui Yue ${ }^{3+}$, Weiwen Shen ${ }^{1}$, Yushan Du${ }^{1}$, Ying Yuan ${ }^{2}$, Xiaofeng Tao ${ }^{2^{*}}$ and Cheuk Ying Tang ${ }^{4}$

\begin{abstract}
Background: Fine needle aspiration biopsy is usually performed to evaluate thyroid lesions. The purpose of this study was to evaluate the usefulness of diffusion weighted imaging to differentiate malignancy of thyroid lesions.

Methods: The study was approved by ethics committee of Shanghai Changzheng Hospital.Forty-two patients, 10 men and 32 women (range: 20-72 years, mean age 42.4 years) with thyroid lesions were included in the study. Routine neck MR and diffusion-weighted MR imaging was performed using multiple b-values. ADC values were computed for the different b-values. Histological results of the thyroidectomy samples were obtained for all the patients. ADC values of benign and malignant thyroid lesions were compared with the pathology results. Logistic regression analysis was used to detect independent parameters for differentiating benign and malignancy of lesions.

Result: Based on the histology results there were 28 benign and 14 malignant cases. The difference of ADC value between benign and malignant thyroid lesions was significant for ADC values obtained using b-values of 0 and $300 \mathrm{~s} /$ $\mathrm{mm}^{2}(p<0.001)$. The ADC values were significantly higher in benign lesions (benign ADC: $2.37 \pm 0.47 \times 10-3 \mathrm{~mm} / \mathrm{s}$ vs. malignant: $\left.1.49 \pm 0.60 \times 10-3 \mathrm{~mm}^{2} / \mathrm{s}\right)$. ADC values obtained with b-values of 0 and $300 \mathrm{~mm}^{2} / \mathrm{s}$ and max nodular diameter was regarded as the two most discriminative parameters for differentiating malignancy. Using the pathology results as a standard reference, area under ROC curve was found to be 0.876 for an ADC cutoff value of $2.17 \times 10-3 \mathrm{~mm}^{2} / \mathrm{s}$ that corresponded to an acquisition with b-values of 0 and $300 \mathrm{~mm}^{2} / \mathrm{s}$.
\end{abstract}

Conclusion: Diffusion-weighted MR imaging is a promising non-invasive method to differentiate malignancy in thyroid lesions.

Keywords: Thyroid lesions, Diffusion weighted imaging, ADC mapping

\section{Background}

Incidence and prevalence of thyroid disease has seen a great increase in recent years. An increasing incidence of thyroid cancer has been reported worldwide [1-3]. The reported incidence of thyroid cancer is up to $0.07 \%$ over the past 30 years [4]. 44,670 new cases and 1,690 deaths were attributed to thyroid cancer in 2010 [5]. Fine needle aspiration biopsy (FNAB) is regarded as the standard reference for diagnosis, but it has been reported before that FNAB results may mimic some other diseases [6-8].

\footnotetext{
* Correspondence: cjr.taoxiaofeng@vip.163.com

${ }^{\dagger}$ Equal contributors

${ }^{2}$ Radiology Department, Shanghai People's Ninth Hospital, Shanghai Jiaotong University, School of Medicine, Shanghai 200011, China

Full list of author information is available at the end of the article
}

Ultrasound is the most common non-invasive and sensitive diagnostic imaging modality for diagnosing thyroid lesions, but there are still no reliable criteria for distinguishing benign from malignant lesions. In addition, it is difficult to access the malignancy of the nodule when it is large or multinodular [9]. Despite tremendous improvement in diagnostic techniques such as ultrasound, radionuclide imaging and CT, there is still a big challenge to have a non-invasive and reliable technique to differentiate benign from malignant lesions. Recent developments in MRI may show that some MR protocols are of diagnostic value for these types of lesions [10]. Routine T1- and T2-weighted MR imaging can provide information on the location and size of thyroid lesions. But these protocols still don't have

\section{Biomed Central}

(c) 2013 Wu et al.; licensee BioMed Central Ltd. This is an Open Access article distributed under the terms of the Creative Commons Attribution License (http://creativecommons.org/licenses/by/2.0), which permits unrestricted use, distribution, and reproduction in any medium, provided the original work is properly cited. 
the specificity for distinguishing benign from malignant nodules or assessing the functional status of these thyroid nodules.

Diffusion-weighted MR imaging (DWI) is an emerging technique for central nervous system (CNS) diseases. DWI is sensitive to changes in the microstructural organization of tissue that may affect water diffusion. It has been used in various forms to evaluate head and neck tumors [11-13]. The Apparent Diffusion Coefficient (ADC) value, a metric obtained from DWI scans, could be a quantitative parameter for distinguishing malignant tumors from benign lesions.

In our current study, we compared ADC values of thyroid lesions with their pathology reports in order to evaluate its usefulness for distinguishing malignancy.

\section{Methods}

\section{Patient selection}

Between July 2007 and Jan 2011, 42 patients (10 male; 32 female) with palpable or ultrasonography determined thyroid nodules were prospectively enrolled and patient consent was obtained. The study was approved by ethics committee of Shanghai Changzheng Hospital. Mean age of those patients was 42.9 yrs (range 20-72 yrs). Routine neck MR imaging and diffusion-weighted MR imaging was performed for each patient. All patients enrolled for this study have been scheduled to have thyroidectomy within 2 weeks. Thyroidectomy was performed afterwards and pathology results were obtained for each patient. Conventional histology revealed that 28 patients had benign lesions, including thyroid adenoma $(\mathrm{N}=20)$, nodular goiter $(\mathrm{N}=5)$, and Hashimoto's thyroiditis $(\mathrm{N}=3)$ while 14 patients were found to have malignant lesions, including thyroid papillary carcinoma $(\mathrm{N}=10)$, follicular thyroid cancer $(\mathrm{N}=3)$, atypical hyperplasia $(\mathrm{N}=1)$. Thyroid lesions less than $1 \mathrm{~cm}$ were excluded because of diagnostic difficulties in evaluation.

\section{MRI protocol}

MR scanning was performed on GE Signa HD 1.5 T MR scanner (GE healthcare, USA). Routine MRI examinations were done with the following parameters: fast spin echo FSE scanning for neck, axial T1WI (TR = $520 \mathrm{~ms}$, $\mathrm{TE}=14 \mathrm{~ms}), \mathrm{T} 2 \mathrm{WI} \quad(\mathrm{TR}=3500 \mathrm{~ms}, \mathrm{TE}=95 \mathrm{~ms})$ and coronal T2WI $(\mathrm{TR}=3000 \mathrm{~ms}, \mathrm{TE}=85 \mathrm{~ms})$. Thickness = $4 \mathrm{~mm}$, Spacing $=1 \mathrm{~mm}$. FOV $=14 \mathrm{~cm} \times 14 \mathrm{~cm}$, Matrix $=$ $320 \times 256, \mathrm{NEX}=4$.

Diffusion Weighted MR Imaging (DWI) was acquired using four different $b$ factors $\left(0,300,500\right.$, and $\left.800 \mathrm{~s} / \mathrm{mm}^{2}\right)$ in a STIR fat suppressed SE-EPI sequence. All DWI scans were acquired with the same parameters:TR $=3000 \mathrm{~ms}$, $\mathrm{TE}=60 \mathrm{~ms}$, Matrix $=96 \times 128$, thickness $-4 \mathrm{~mm}$ and 6 averages were obtained. Total scanning time of about 10 minutes for the DWI scan.

\section{Image post processing}

Using the post processing software Functool from GE, ADC maps were automatically generated for each of the b factors. ADC values were extracted from ADC maps. Circular ROIs (regions of interests)with an area of $1 \mathrm{~cm}^{2}$ were carefully placed on the lesions (areas of necrosis, hemorrhage, calcium and cyst formation were excluded). ROIs were placed on one ADC map and then propagated to the other 2 by two experienced radiologists blinded to each other's delineations. To minimize noise, each radiologist made three measurements for each lesion and the mean $\mathrm{ADC}$ value was recorded. Interrater reliability of the ROIs ADC values had ICC $>0.87$. Final $\mathrm{ADC}$ value was determined by the mean values from two raters.

\section{Analysis}

ADC maps were computed for each of the b-values used in the DWI protocol. This was done automatically for the b-factors of $300 \mathrm{~s} / \mathrm{mm}^{2}, 500 \mathrm{~s} / \mathrm{mm}^{2}$ and $800 \mathrm{~s} / \mathrm{mm}^{2}$. ADC values were extracted from the ADC maps. Statistical analysis was performed using the Statistical Package for the Social Sciences for Windows (SPSS, Chicago, Ill). All ADC data were recorded in Mean $\pm \mathrm{SD}\left(\times 10^{-3} \mathrm{~mm}^{2} / \mathrm{s}\right)$ form. Mann-Whitney U-test was performed to compare the quantitative ADC value of benign and malignant thyroid nodules. A value of $\mathrm{p}<.05$ was considered significant. In addition, receiver operating characteristic (ROC) curve was constructed to determine a cutoff value for differentiating benign and malignant thyroid lesions. Receiver operating characteristic (ROC) curves were constructed and areas under the ROC curve (Az) were computed using MedCalc version 10.2.0.0 (http://www.medcalc.com/).

\section{Results}

MRI results revealed that among those 42 cases of lesions in the thyroid, 10 were located in the left lobe of thyroid gland, 16 of were in the right lobes, 14 had lesions on both lobes and 2 lesions were in thyroid isthmus. For those with lesions on both lobes, one was randomly chosen (matched with pathology) for ADC measurements. Mean major diameter of the benign and malignant lesions were $3.63 \mathrm{~cm}$ and $2.55 \mathrm{~cm}$ respectively. Historical findings confirmed there were 28 benign cases and 14 malignant lesions. Independent of malignancy, thyroid lesions occurred more frequently in female than in male (Table 1).

\section{Parameters for differentiating lesion malignancy}

Using histology results to group the ADC values we found that the ADC values were significantly different $(\mathrm{p}<0.001)$ between benign and malignant lesions for the ADC values computed for $b=300 \mathrm{~s} / \mathrm{mm}^{2}$. Mean ADC value for benign group (Figure 1) is much higher than that for malignant group (Figure 2), with the values of 
Table 1 Historical results and patient characteristics

\begin{tabular}{ccccc}
\hline Histological categories & Number (\%) & Mean age (yrs) & Female (\%) & Mean maximum diameter (cm) \\
\hline Benign & $28(66.7)$ & 41.7 & $21(75)$ & $3.63 \pm 0.37$ \\
Thyroid adenoma & $20(47.6)$ & 42.6 & $15(53.6)$ & $3.53 \pm 0.25$ \\
Nodular goiter & $6(14.3)$ & 39.3 & $5(17.9)$ & $3.95 \pm 0.65$ \\
Hashimoto's thyroiditis & $2(4.8)$ & 45.6 & $1(3.6)$ & $3.71 \pm 0.11$ \\
Malignant & $14(33.3)$ & 45.3 & $11(78.6)$ & $2.55 \pm 0.41$ \\
Thyroid papillary carcinoma & $11(26.2)$ & 41.9 & $1(7.1)$ & $2.73 \pm 0.54$ \\
Follicular thyroid cancer & $2(4.8)$ & 49.1 & $1(7.1)$ & $1.7 \pm 0.13$ \\
Atypical hyperplasia & $1(2.4)$ & 45 & & 2.3 \\
\hline
\end{tabular}

$2.37 \pm 0.47 \times 10^{-3} \mathrm{~mm}^{2} / \mathrm{s}$ and $1.49 \pm 0.60 \times 10-3 \mathrm{~mm}^{2} / \mathrm{s}$ respectively. However, no significant difference was observed between the two groups for the ADC values obtained using $\mathrm{b}=500 \mathrm{~s} / \mathrm{mm}^{2}$ or $800 \mathrm{~s} / \mathrm{mm}^{2}$ (Table 2). Sensitivity, specificity and AUC were also compared among three different b-factors. Highest sensitivity and AUC was observed at b-factor of $300 \mathrm{~s} / \mathrm{mm}^{2}$ (Table 3). We also selected four variables (age, sex, ADC value, max nodular diameter) as independent variables to perform logistic regression analysis. Odds ratios (and 95\% CIs) based on the logistic regression model were also calculated. The results obtained from the logistic regression model showed that ADC values obtained with $\mathrm{a} b$ factor of $300 \mathrm{~s} / \mathrm{mm}^{2}(\mathrm{OR}=4.76, \mathrm{p}<0.001)$ and $\max$ nodular diameter $(\mathrm{OR}=3.22, \mathrm{p}=0.034)$ were the two most discriminative independent variables for differentiating between benign and malignant lesions (Table 2).

\section{ROC analysis}

Furthermore, a Receiver Operator Characteristic (ROC) curve was computed for the ADC values obtained from DWI scans using $b=300 \mathrm{~s} / \mathrm{mm}^{2}$. We determined a cutoff point from the ROC curve that would differentiate benign from malignant lesions. The area under the ROC curve was 0.876 . When we selected a cutoff $\mathrm{ADC}$ value of $2.17 \times 10^{-3} \mathrm{~mm}^{2} / \mathrm{s}$, sensitivity and specificity was found to be $76.5 \%$ and $100 \%$ respectively (Figure 3 ).

\section{Discussion}

To date, only a few studies have focused on the use of DWI in evaluating thyroid disease. Tezuka [14] et al. used both routine T1WI, T2WI and DWI techniques to diagnose thyroid diseases (Graves' disease, sub-acute thyroiditis and Hashimoto's disease). Although no obvious difference using T1WI and T2WI were detected

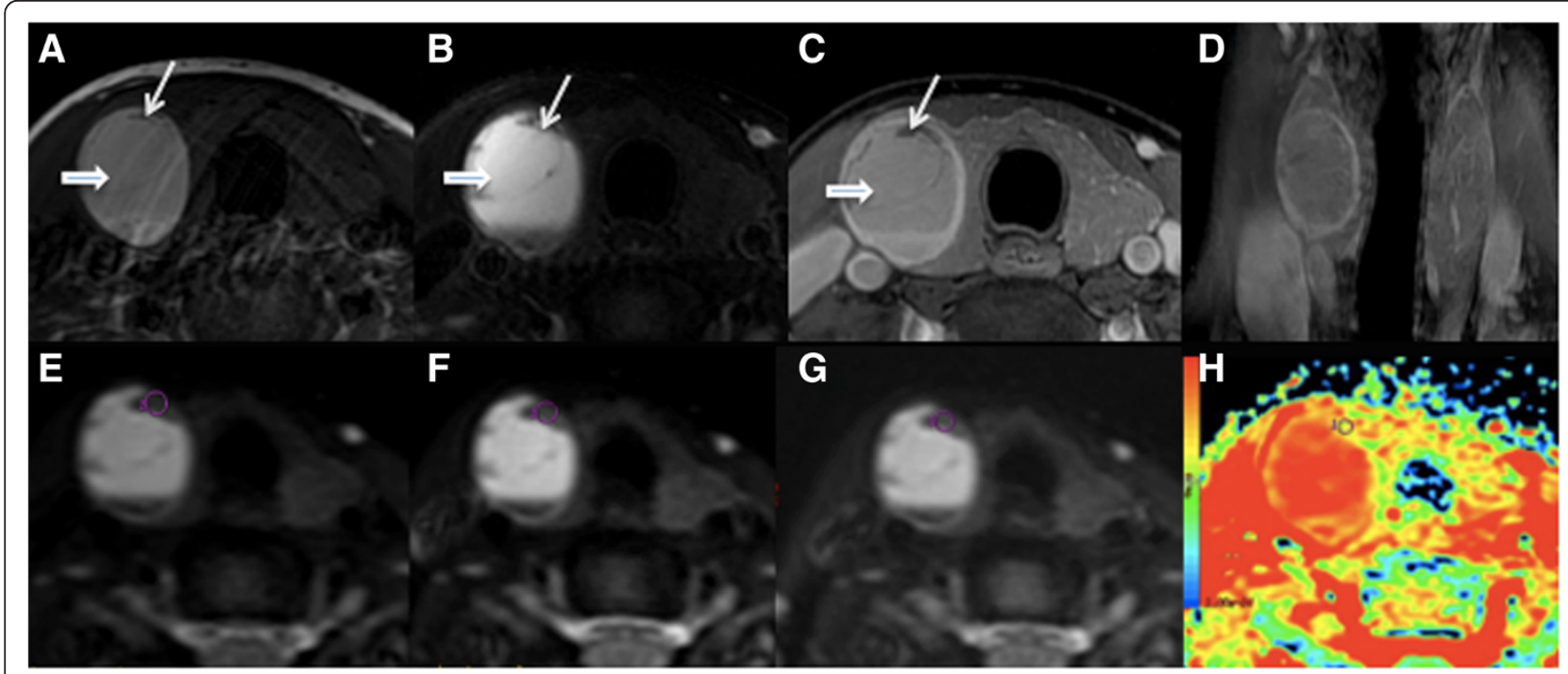

Figure 1 A 44-year-female patient with right lobe thyroid adenoma; (A-C) Non-contrast and contrast transversal images showed a hemorrhage in the right lobe (short thick arrow). Long thin arrow shows the lesion. (D) Coronal images showed the well-circumscribed lesion with homogenous enhancement. (E-G) showed ADC value obtained from ADC map with b factors of 0, 300, 500 and $800 \mathrm{~s} / \mathrm{mm}^{2}$, respectively. ROls were placed in the lesion at right upper area to avoid the hemorrhage area. (H) ADC map generated at b-factor of $300 \mathrm{~s} / \mathrm{mm}^{2}$. 


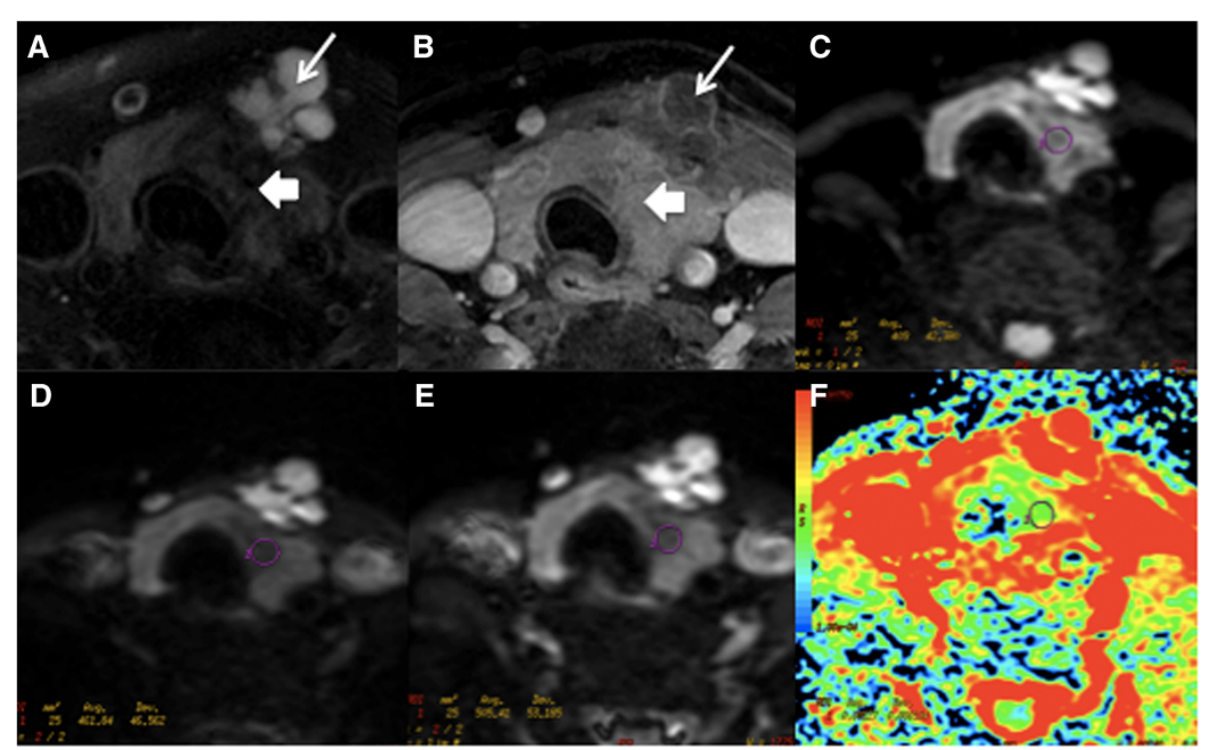

Figure 2 A-36-year-female patient with thyroid papillary carcinoma at left lobe and isthmuses is shown. (A-B) Non-contrast and contrast transversal images showed abnormal signal at left lobe and isthmus with multiple cysts (long arrows). (C-E) showed ADC value measured from ADC map with b factors of 300,500 and $800 \mathrm{~s} / \mathrm{mm}^{2}$, respectively. (F) ADC map generated at b-factor of $300 \mathrm{~s} / \mathrm{mm}^{2}$.

on those thyroid diseases, ADC values of thyroid lesions of Graves' disease was found much higher than that of sub-acute thyroiditis or Hashimoto's disease. An ADC value of $1.82 \times 10^{-3} \mathrm{~mm}^{2} / \mathrm{s}$ was suggested as the cutoff point to diagnose Graves' disease (Sensitivity $75 \%$ and Specificity $80 \%$ ). DWI has emerged as a potential new noninvasive technique that can provide more quantitative information on thyroid lesions and help make clinical differential diagnosis.

\section{MR technique}

Diffusion weighted MR imaging can be done with single shot or multi-shot. Wang and Adel Razek [11,15] applied single-shot multisection echo-planar technique, using b values of 0,500 and $1000 \mathrm{~s} / \mathrm{mm}^{2}$ respectively. This sequence is characterized by a train of gradient echoes with a short echo time image and good signal-to-noise ratio
(SNR), but it was associated with more magnetic susceptibility artifacts. Tezuka [14] et al., used a fast spin echo sequence for diffusion-weighted imaging to avoid magnetic susceptibility artifacts, but not only was the imaging time increased but the SNR was also decreased.

In this study, we used a STIR (Short TI Inversion Recovery) fat suppressed SE EPI (Spin-Echo Echo Planar Imaging) sequence. STIR is a very robust fat suppression technique with minimal artifacts. It has low sensitivity to magnetic field inhomogeneities or susceptibility effects and thus improves image quality. It also produces a slight T1-weighted background suppression but does not affect the accuracy of the images. EPI is the fastest acquisition method and it only requires $30 \mathrm{~ms} \sim 100 \mathrm{~ms}$ to collect one image which helps in reducing most physiological movement artifacts [16]. Furthermore, short TE time of $60 \mathrm{~ms}$ was used to reduce T2* effect in our study. Thus,

Table 2 Association between parameters and disease property

\begin{tabular}{|c|c|c|c|c|c|}
\hline & Benign group $(n=28)$ & Malignant group $(n=14)$ & $P_{\text {value }}^{*}$ & OR & $95 \% \mathrm{Cl}$ \\
\hline Age (yrs) & 41.7 & 45.3 & 0.79 & 0.62 & $0.26,2.59$ \\
\hline Sex (Female \%) & $21(75)$ & $11(78.6)$ & 0.61 & 0.50 & $0.16,1.54$ \\
\hline Max nodular diameter $(\mathrm{cm})$ & $3.63 \pm 0.37$ & $2.55 \pm 0.41$ & 0.034 & 3.22 & $1.27,6.76$ \\
\hline \multicolumn{6}{|l|}{$A D C$ values $\left[\mathrm{mm}^{2} / \mathrm{s}\right]$} \\
\hline$b-0,300$ & $2.37 \pm 0.47$ & $1.49 \pm 0.60$ & $<0.001$ & 4.76 & $1.56,9.89$ \\
\hline b-0,500 & $1.87 \pm 0.25$ & $1.61 \pm 0.45$ & 0.138 & 1.01 & $0.3,1.81$ \\
\hline$b-0,800$ & $1.68 \pm 0.25$ & $1.41 \pm 0.34$ & 0.059 & 0.091 & $0.03,0.73$ \\
\hline
\end{tabular}

*p value reflected from logistic analysis. 
Table 3 Sensitivity, specificity and AUC of the use of mean ADC value as calculated based on 3 different b-values for differentiating benign from malignant thyroid lesions

\begin{tabular}{cccc}
\hline & $\mathbf{b}=\mathbf{0 , 3 0 0}$ & $\mathbf{b}=\mathbf{0 , 5 0 0}$ & $\mathbf{b}=\mathbf{0 , 8 0 0}$ \\
\hline AUC & $0.88(0.77-0.97)$ & $0.63(0.47-0.77)$ & $0.63(0.46-0.77)$ \\
Criterion $\left[10^{-3} \mathrm{~mm}^{2} / \mathrm{s}\right]$ & $>2.17$ & $>1.74$ & $>1.65$ \\
Sensitivity \% & 76.5 & 67.9 & 53.6 \\
Specificity \% & 100 & 64.3 & 71.4 \\
\hline
\end{tabular}

using this sequence we were able to obtain more accurate tissue ADC values.

The b-factor in the DWI was an important factor for image quality. We obtained diffusion-weighted MR images with different $\mathrm{b}$ factors simultaneously to avoid misregistration in computing the different ADC values. Higher $\mathrm{b}$-values produce more diffusion weighting and therefore higher contrast between thyroid lesions and normal tissue. However, higher $\mathrm{b}$ value also leads to increased signal attenuation and usually required more averages to compensate for the SNR. Higher b-values also produce more susceptibility distortions and could increase the noise in the DWI images because the distortions are different depending on the gradient directions. In addition, different tumors within different organs or tissues may be more sensitive to different b-values. In this study, we applied an SE EPI diffusion imaging with b-factors of 300, 500 and $800 \mathrm{~s} / \mathrm{mm}^{2}$. Our results showed that a b-factor of 300 $\mathrm{s} / \mathrm{mm}^{2}$ was sufficient to obtain high quality ADC values

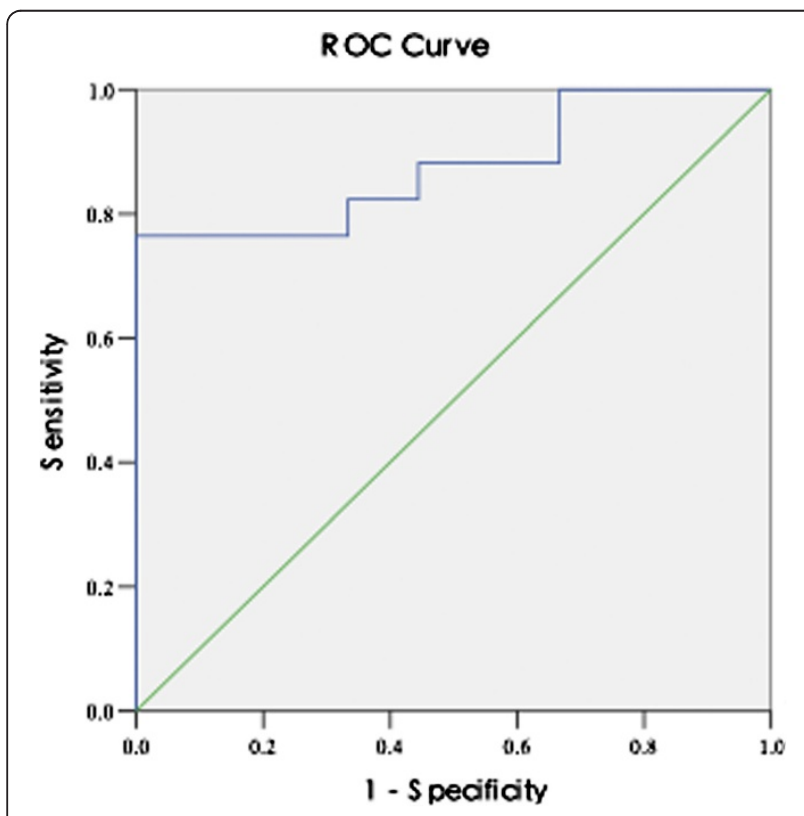

Figure 3 Receiver operating characteristic (ROC) curve with the $A D C$ value (computed from DWI with $b=0$ and $300 \mathrm{~s} / \mathrm{mm}^{2}$ ) used for differentiating benign from malignant thyroid lesions. and it was also the one with had a high sensitivity and accuracy for differentiating benign and malignancy. These results are consistent with Bozgeyik's [17] results.

\section{Pathological mechanism of ADC values in thyroid lesions}

In our study we were able to use the most reliable reference for malignancy which was based on the histopathology of a thyroidectomy. Our results showed a significant difference in the ADC value between benign and malignant thyroid lesions, with ADC values in benign lesions being higher than malignant lesions $\left(2.37 \pm 0.47 \times 10^{-3} \mathrm{~mm}^{2} / \mathrm{s}\right.$ vs. $1.49 \pm 0.60 \times 10^{-3} \mathrm{~mm}^{2} / \mathrm{s}$ ) at the $\mathrm{b}$ factor of $300 \mathrm{~s} / \mathrm{mm}^{2}$. These results are consistent with other similar studies [17-19]. Diffusion weighted imaging provides more information on the microstructure of tissues and their physiological processes. Changes in the distributions of intracellular organelles and macromolecules in the tissues affect the random motion of water protons. ADC values of tissues vary according to its cellularity and histopathology. Anderson [20]'s study showed that malignant lesions in thyroid glands was characterized by compact cellularity which increased the nucleocytecytoplasmic ratio (NCR). These microscopic pathological changes results in the reduction of extracellular space which limits diffusional motion of water protons. Reduced ADC values are presented as high signal on DWI sequences.

Abdel's [21] study demonstrated that when selecting ADC value of $0.98 \times 1.0^{-3} \mathrm{~mm}^{2} / \mathrm{s}$ as a cutoff point to differentiate benign and malignant lesions, the sensitivity and specificity was $97.5 \%$ and $91.7 \%$, respectively. The accuracy was up to $98.9 \%$. In this study a single pair of bvalues $\left(0,1000 \mathrm{~s} / \mathrm{mm}^{2}\right)$ were studied. Although we also looked at the ADC values obtained using higher b-values $\left(0,800 \mathrm{~s} / \mathrm{mm}^{2}\right)$, we did not detect any useful threshold at the higher b-values. Abdel's study included a variety of cervical lymph nodes whereas our study focused on the thyroid. Lesion heterogeneity might have accounted for the differences in sensitivity. It was also suggested that the $A D C$ values of benign thyroid nodule may vary according to the complex composition within the nodule (colloid, tiny necrosis and cystic change, hemorrhage, fibrosis and calcium). ADC values were highest in thyroid cysts since it contained colloid cyst made of serous or concentrated thyroglobulin. Conversely, increasing NCR and grit-like calcification mainly lead to a decrease of ADC values in papillary thyroid carcinoma.

Bozgeyik [17] also studied thyroid nodules with DWI at lower b-values. Bozgeyik [17] also found that a b-factor of $300 \mathrm{~s} / \mathrm{mm}^{2}$ was the most useful for differentiating benign or malignant lesions. In Bozgeyik's study 3 b-value pairs were used to computed the $\operatorname{ADC}\left(0,100 \mathrm{~s} / \mathrm{mm}^{2}\right),(0$, $\left.200 \mathrm{~s} / \mathrm{mm}^{2}\right)$ and $\left(0,300 \mathrm{~s} / \mathrm{mm}^{2}\right)$. We expanded on these results and investigated $\mathrm{ADC}$ values obtained using larger 
b-values. Our sample includes about 33\% malignancy rates whereas in Bozgevik's study less than $5 \%$ of the nodules were malignant. In their study, malignancy was determined using FNAB whereas in our study we have used pathology results from thyroidectomy, a more accurate reference. Erdem [19] also suggested that tiny calcification was closely correlated with the reduction of ADC values in thyroid malignancy.

However, in Weidekamm's [22,23] study, the opposite result was reported. ADC values were predominantly higher in malignant thyroid nodules than that in benign nodules with values equal or more than $2.25 \times 10^{-3} \mathrm{~mm}^{2} / \mathrm{s}$. The higher ADC values may be due to the over production of thyroprotein follicle in malignant thyroid nodules which do not restrict the diffusion of the water protons.

ADC values has been used as a quantitative parameter for differentiating benign and malignant lesions [24]. The ADC values depend on many factors such as tissue microstructure, necrosis, presence of macromolecules and also perfusion phenomenon. When compared to benign lesions, abnormal blood perfusion is more prevalent in malignant lesions and ADC values will be affected by both blood perfusion and extracellular space. Unlike some other studies [17], in the current study, we chose three different $b$ factors all over $300 \mathrm{~s} / \mathrm{mm}^{2}$ since ADC value would better reflect the true water diffusion in the tissue with larger $b$ factors. However, there was no significant difference in ADC value between benign and malignant lesions when the b factors were $500 \mathrm{~s} / \mathrm{mm}^{2}$ and 800 $\mathrm{s} / \mathrm{mm}^{2}$. Delormere [25] demonstrated that malignant lesions usually do not have a complete basal membrane of blood vessel which enhances the molecular exchange in the capillary bed. ADC values could be influenced by both blood perfusion and extracellular space. In thyroid malignancy, increased blood perfusion increases the apparent speed of the diffusing water protons while narrow extracellular space will restrict its movement. Although at higher b-values the sensitivity to perfusion is reduced, our results show no difference in ADC values between benign and malignant groups with b-values of $500 \mathrm{~s} / \mathrm{mm}^{2}$ or $800 \mathrm{~s} / \mathrm{mm}^{2}$. The ADC values obtained using the lower b-value of $300 \mathrm{~s} / \mathrm{mm}^{2}$ is most likely affected by both perfusion and diffusion effects. It is possible that the discriminatory effect of this lower $300 \mathrm{~s} / \mathrm{mm}^{2}$ reflect a combination of altered vascularity and changes in cellular composition that characterizes malignancy.

Fine-needle aspiration biopsy (FNAB) cytology can give 4 different results: benign, malignant, suspicious and nondiagnostic. No definite diagnosis is possible for the two categories of suspicious and non-diagnostic. Although FNAB is commonly used to screen for malignancy, a unique approach in this study is that we used histological results from thyroidectomy as the standard reference for distinguishing benign and malignant lesions. Based on final histological results, we were able to obtain a definite diagnosis of each lesion. ROC curves and cut off point for b-values are more accurate in this approach.

There are still some limitations in this study. First, the relatively small number $(33.3 \%)$ of the malignant nodules somehow limits the statistical power. This study needs to be expanded further with a larger number of patients. B-values should be extended to lower values and higher ranges to verify that $300 \mathrm{~s} / \mathrm{mm}^{2}$ is an optimal value. Second, thyroid nodules less than $10 \mathrm{~mm}$ were not included in this study. Improvement in the software of diffusion-weighted MR imaging will help in the detection of smaller lesions in future studies. Third, we have not studied the correlation of ADC values with subtypes of thyroid lesions such as cysts from hemorrhagic nodules because of the small number which would reduce its statistical power.

\section{Conclusion}

In conclusion, diffusion-weighted MR imaging is a relatively new and non-invasive approach to assess thyroid lesions. ADC values seem to be able to differentiate benign from malignant thyroid disease. Further studies should be performed to expand the utility of DWI in thyroid lesions.

\section{Abbreviations}

DWI: Diffusion-weighted imaging; ADC: Apparent diffusion coefficient; T1WI: T1 weighted imaging; T2WI: T2 weighted imaging; FNAB: Fine needle aspiration biopsy; ICC: Intraclass correlation coefficient; ROC: Receiver operating characteristic.

\section{Competing interests}

The authors declare that they have no competing interests.

\section{Authors' contributions}

YW collect data from patients and drafted the manuscript. XY participated in the sequence alignment. WS participated in the sequence alignment. YD did postprocessing and measurement on images. YY did postprocessing and measurement on images. XT conceived of the study, and participated in its design and coordination. CT participated in the design of the study and performed the statistical analysis. All authors read and approved the final manuscript.

\section{Acknowledgments}

The manuscript was supported by grant of National Natural Science Foundation of China (Dr. Xiaofeng Tao No. 81272802) and Key scientific and innovation grant of Education Commission of Shanghai (Dr. Yingwei Wu No. 12ZZO28). Outstanding academic leader of Shanghai Science and Technology Committee of Dr. Xiaofeng Tao (Grant No. 13XD1402400).

\section{Author details}

${ }^{1}$ Radiology Department, East Hospital, Tongji University, school of Medicine, Shanghai 200120, China. ${ }^{2}$ Radiology Department, Shanghai People's Ninth Hospital, Shanghai Jiaotong University, School of Medicine, Shanghai 200011, China. ${ }^{3}$ Radiology Department, Shanghai Changzheng Hospital, Shanghai, China. ${ }^{4}$ Radiology Department, Mount Sinai School of Medicine, New York 10029, USA.

Received: 6 November 2012 Accepted: 19 July 2013

Published: 30 July 2013 


\section{References}

1. Wong CK, Wheeler MH: Thyroid nodules: rational management. World J Surg 2000, 24:934-941.

2. Brander A, Viikinkoski P, Nickels J, Kivisaari L: Thyroid gland: US screening in a random adult population. Radiology 1991, 181:683-687.

3. Davies $L$, Welch HG: Increasing incidence of thyroid cancer in the United States, 1973-2002. JAMA 2006, 295:2164-2167.

4. Mazzaferri EL, Harmer C, Mallick UK, Kendall-Taylor P: Practical management of thyroid cancer: a multidisciplinary approach. London: Springer; 2006.

5. Jemal A, Siegel R, Xu J, Ward E: Cancer statistics, 2010. CA Cancer J Clin 2010, 60:277-300.

6. Bakshi NA, Mansoor I, Jones BA: Analysis of inconclusive fine-needle aspiration of thyroid follicular lesions. Endocr Pathol 2003, 14:167-175.

7. Koike E, Yamashita H, Noguchi S, Murakami T, Ohshima A, et al: Effect of combining ultrasonography and ultrasound-guided fine-needle aspiration biopsy findings for the diagnosis of thyroid nodules. Eur J Surg 2001, 167:656-661.

8. McCoy KL, Jabbour N, Ogilvie JB, Ohori NP, Carty SE, et al: The incidence of cancer and rate of false-negative cytology in thyroid nodules greater than or equal to $4 \mathrm{~cm}$ in size. Surgery 2007, 142:837-844. discussion 844 e831-833.

9. Solbiati L, Osti V, Cova L, Tonolini M: Ultrasound of thyroid, parathyroid glands and neck lymph nodes. Eur Radiol 2001, 11:2411-2424.

10. Kiyosue $H$, Miyake $H$, Komatsu E, Mori H: MRI of cervical masses of thymic origin. J Comput Assist Tomogr 1994, 18:206-208.

11. Wang J, Takashima S, Takayama F, Kawakami S, Saito A, et al: Head and neck lesions: characterization with diffusion-weighted echo-planar MR imaging. Radiology 2001, 220:621-630.

12. Eida S, Sumi M, Sakihama N, Takahashi H, Nakamura T: Apparent diffusion coefficient mapping of salivary gland tumors: prediction of the benignancy and malignancy. AJNR Am J Neuroradiol 2007, 28:116-121.

13. King $\mathrm{CH}$, Higa AT, Culjat MO, Han SH, Bisley JW, et al: A pneumatic haptic feedback actuator array for robotic surgery or simulation. Stud Health Technol Inform 2007, 125:217-222.

14. Tezuka M, Murata Y, Ishida R, Ohashi I, Hirata Y, et al: MR imaging of the thyroid: correlation between apparent diffusion coefficient and thyroid gland scintigraphy. Journal of magnetic resonance imaging: JMRI 2003, 17:163-169.

15. Abdel Razek AA, Soliman NY, Elkhamary S, Alsharaway MK, Tawfik A: Role of diffusion-weighted MR imaging in cervical lymphadenopathy. Eur Radiol 2006, 16:1468-1477.

16. Murtz P, Krautmacher C, Traber F, Gieseke J, Schild HH, et al: Diffusion-weighted whole-body MR imaging with background body signal suppression: a feasibility study at 3.0 Tesla. Eur Radiol 2007, 17:3031-3037.

17. Bozgeyik Z, Coskun S, Dagli AF, Ozkan Y, Sahpaz F, et al: Diffusion-weighted MR imaging of thyroid nodules. Neuroradiology 2009, 51:193-198.

18. Razek AA, Sadek AG, Kombar OR, Elmahdy TE, Nada N: Role of apparent diffusion coefficient values in differentiation between malignant and benign solitary thyroid nodules. AJNR Am J Neuroradio/ 2008, 29:563-568.

19. Erdem G, Erdem T, Muammer H, Mutlu DY, Firat AK, et al: Diffusion-weighted images differentiate benign from malignant thyroid nodules. Journal of magnetic resonance imaging: JMRI 2010, 31:94-100.

20. Anderson JR (Ed): Muir'sTextbook of Pathology. 13th edition. London: Edward Arnold; 1992

21. Abdel Razek AA, Soliman NY, Elkhamary S, Alsharaway MK, Tawfik A: Role of diffusion-weighted MR imaging in cervical lymphadenopathy. Eur Radiol 2006, 16:1468-1477.

22. Schueller-Weidekamm C, Kaserer K, Schueller G, Scheuba C, Ringl H, et al Can quantitative diffusion-weighted MR imaging differentiate benign and malignant cold thyroid nodules? Initial results in 25 patients. AJNR Am J Neuroradiol 2009, 30:417-422.

23. Schueller-Weidekamm C, Schueller G, Kaserer K, Scheuba C, Ringl H, et al Diagnostic value of sonography, ultrasound-guided fine-needle aspiration cytology, and diffusion-weighted MRI in the characterization of cold thyroid nodules. Eur J Radiol 2010, 73:538-544.

24. Herneth AM, Guccione S, Bednarski M: Apparent diffusion coefficient: a quantitative parameter for in vivo tumor characterization. Eur J Radiol 2003, 45:208-213.

25. Delorme $\mathrm{S}, \mathrm{Knopp} \mathrm{MV}$ : Non-invasive vascular imaging: assessing tumour vascularity. Eur Radiol 1998, 8:517-527.

doi:10.1186/1471-2342-13-23

Cite this article as: Wu et al:: Diagnostic value of diffusion-weighted MR imaging in thyroid disease: application in differentiating benign from malignant disease. BMC Medical Imaging 2013 13:23.

\section{Submit your next manuscript to BioMed Central and take full advantage of:}

- Convenient online submission

- Thorough peer review

- No space constraints or color figure charges

- Immediate publication on acceptance

- Inclusion in PubMed, CAS, Scopus and Google Scholar

- Research which is freely available for redistribution

Submit your manuscript at www.biomedcentral.com/submit
C Biomed Central 